EPiC Series in Computing
Volume 69, 2020, Pages 345-354
Proceedings of 35th International Confer-
ence on Computers and Their Applications

\title{
Channel-Matched OFDM (CM-OFDM) for Enhanced Performance of Multiuser Systems Operating in Underwater Acoustic Channels
}

\author{
Said E. El-Khamy ${ }^{1}$ and Dalia I. Mohamed ${ }^{2}$ \\ ${ }^{12}$ Dept. of Electrical Engineering, Faculty of Engineering. Alexandria University, Alexandria \\ 21544, Egypt. \\ elkhamyeieee.org and dalia.ibrahimealexu.edu.eg
}

\begin{abstract}
The design of multiuser OFDM underwater acoustic (UWA) communication systems is very challenging due to the time-varying and frequency selective fading of UWA channels. The key for ensuring a reliable optimum transmission for every user is the suitable resource assignment for each. This paper proposes a new adaptive channelmatched OFDM scheme (CM-OFDM) to overcome the performance degradation caused by the frequency selective fading across the OFDM subcarriers. In the proposed scheme, the OFDM subcarriers are sorted depending on their corresponding channel gains. Then the resource assignment to the different users is accomplished according to their required quality of service (QOS). The user with the highest QOS is assigned the best subcarriers and the other users are similarly assigned the remaining subcarriers. This optimized resource assignment technique guarantees enhanced performance with no need of increasing the transmitted power or changing the modulation schemes. The performance of the proposed technique is investigated and is compared with uniform and random subcarriers' assignment methods used for multiuser OFDM systems. The simulations are performed for a multipath frequency selective UWA channel model. The simulation results clearly show the advantages of CM-OFDM scheme for the users with high QOS on the expense of a slight degradation in the performance for the other user with lower QOS.
\end{abstract}




\section{Introduction}

Underwater acoustic channels (UWAC) are one of the most challenging transmission media. Sound propagation in Underwater channel is characterized by major factors: attenuation, time-varying multipath propagation, and low speed of sound. The multipath formation is due to sound reflection at the surface, the bottom of the sea, or any objects that strikes the wave, and sound refraction in the water. Delay spreading causes inter symbol interference (ISI). The time-varying multipath propagation results in frequency-selective fading. In UWAC this transmission loss depends on the signal frequency. Water variations and environmental events may contribute to changes in the channel response through transmission. Doppler shift is one of these facts that couldn't be ignored when studying acoustic signal propagation in UWC. The Doppler effect may appear due to the motion of the transmitter or the receiver, and causes a frequency shifting. As the speed of sound is low compared to that of the propagated wave, this motion-induced Doppler shift can be extreme compared to the operating frequency. The Bit-ErrorRate (BER) performance of the UWA systems degrades as a result of time-varying multipath propagation, the Doppler shifting and other water properties such as temperature, water salinity and attenuation. [1-3].

Orthogonal frequency-division multiplexing (OFDM) appears as suitable and promising transmission system alternative to single-carrier systems for UWA communications. The reason of wide use of OFDM in UWAC, is its robustness to channels that show long delay spreads and frequency selectivity. As, it decomposes a frequency- selective channel into a set of independent and inter-symbol interference (ISI) free sub-channels [1], [4]. So, if a part of the spectrum suffers from strong fading or dispersion, only few subcarriers will be affected. Unlike a single carrier system, where the entire link will get failed if signal gets fade.

In OFDM UWA communications, some subcarriers may be subjected to a deep attenuation or dispersion. Data transmitted over these subcarriers is exposed to high probability of error so the overall throughput of the system is affected [5]. Adaptive OFDM improves overall system performance by adapting transmission scheme following channel dispersion. We investigate an adaptive scheme to mitigate deep fading effect by avoiding transmission through subcarriers corresponding to high dispersion. So, the transmitted power and modulation doesn't change, only the pattern of subcarriers used in transmission will match the channel conditions.

We may extend the work for a multiuser OFDM system. In channel-matched single user OFDM system, a group of subcarriers is selected and the others remain idle. We can benefit of these unused subcarriers in a transmission of another system. Specially, in multiuser system, where users have different priorities or requires different QOS.

\section{Channel-Matched OFDM}

Orthogonal Frequency Division Multiplexing (OFDM) is a multicarrier transmission. The available bandwidth is divided into a group of orthogonal frequency sub-channels. Each sub-channel is modulated by a separate data symbol and then the sub- channels are frequency multiplexed. In narrowband interference or fading, only few subcarriers are going to be affected. Unlike a single carrier system, where the entire link will fail. [4], [5]. This is the main reason to widely use OFDM in frequency selective environment such as UWAC.

However, the transmission of OFDM signals in Underwater channels is challenging because of the dispersive channel nature, where some subcarriers may be subjected to a deep fading. Adaptive transmission may be used to mitigate heavy fading effect and improve the system performance by matching transmission parameters following the channel characteristics and variations [7]. 
We propose an adaptive OFDM transmission scheme, channel-matched OFDM (CM-OFDM), that increases the throughput. The target is avoiding transmission over subcarriers which suffer from bad channel conditions as it is expected to result in high probability of error. In the proposed algorithm number of used subcarriers for data transmission $\left(N_{o n}\right)$ is fixed, but their frequencies will vary based on channel frequency response to match the channel dispersion profile. As the transmission scheme is adapted to the channel characteristics, thus accurate channel estimation at the receiver with reliable feedback path to the transmitter is required. Pilots are transmitted in the OFDM block at fixed frequency sub-bands, at the receiver these pilots help in channel estimation and equalization. The channel state information (CSI) is sent to the transmitter to select the $N_{\text {on }}$ sub-bands to carry data symbols in the next frame. The selection of the subcarriers' pattern is such that the used frequency slots are concentrated in the frequency sub-bands, characterized by high channel-transmission coefficients. Thus, transmission in the frequency slots, which correspond to relatively high attenuation or bad transmission, is prevented [8].

The proposed CM- OFDM approach insures a reliable transmission. But comparing it with a conventional OFDM system the uses all subcarriers available, the new scheme works at a lower rate as only a subset of subcarriers is used in transmission. To increase the rate to be as OFDM, data can be modulated to higher order making use of the good quality of the channel that will support the high order of modulation without a need to increase the transmitted power.

\section{CM-OFDM System Model}

\subsection{Selection of Channel-matched Subcarriers}

The key in channel-matched OFDM system is the accurate matching of picked frequency pattern to the channel dispersion. The channel profile is presented by transmission coefficient for each of the OFDM subcarriers $|H(f)|^{2}$. The selection is such that used frequency slots are characterized by high channel-transmission coefficients and frequency slots corresponding to relatively high attenuation or bad transmission are prevented. Mathematically, in OFDM, the subcarriers are uniformly distributed within the available bandwidth $\left(B_{o f d m}\right)$. But in channel-matched approach, the selected subcarrier's pattern will be treated as non-uniformly distributed random variable, $F$. Its probability density function (pdf) will depends on the channel transmission coefficients [8], and can be defined by,

$$
p_{F}(f)=C|H(f)|^{2}, f \in B_{o f d m}
$$

where $C$ is a constant to ensure that the integration of $p_{x}(f)$ is unity. The cumulative distribution function (CDF) of $\mathrm{F}$ is,

$$
P_{f}(x)=\int_{f_{\min }}^{x} p_{F}(f) d x
$$

where $f_{\min }$ is the lower frequency edge of the OFDM system bandwidth.

One way to determine the selected frequency pattern, is to solve the Inverse transform sampling problem. This is a method for generating random variable from any probability distribution by using its inverse cumulative distribution. The task is to generate a random variable(rv) F with cumulative distribution function $(\mathrm{CDF}), P_{f}(x)$, described in (2).

The inverse algorithm is simple, first we generate a uniform random variable, $\mathrm{X} \sim \mathrm{Unif}(0,1)$, then we apply the inverse transformation, 
Channel-Matched OFDM (CM-OFDM) for Enhanced Performance of ... S. El-Khamy and D. Ibrahim

$$
F=P_{f}^{-1}(x)
$$

Where $P_{f}^{-1}(x)$, is the inverse of CDF of F, $X$ is rv that, in our work, represents a uniformly distributed frequency slots and $F$ represents the selected channel-matched subcarrier pattern [8].

\subsection{System Model}

The OFDM modulator is the inverse fast Fourier transform (IFFT) matrix, $G$, where each row represents an OFDM carrier defined by,

$$
g_{k}[n]=e^{\frac{j 2 \pi k n}{N}} \quad, \mathrm{k}, \mathrm{n}=0, \ldots, \mathrm{N}-1
$$

Where $g_{k}[n]$ is the carrier in the OFDM modulator, $k$ and $n$ represent the frequency index and time index of subcarriers, respectively and $N$ is the sampling time and the total number of orthogonal subcarriers in OFDM [9].

To generate subcarriers of the proposed channel matched OFDM, we select a subset of $K(K<N)$ OFDM subcarriers, and rows which represents non-selected subcarriers, in $G$ matrix will be set to zero to obtain a new matrix defined by,

$$
G_{\text {matched }}[k,:]=\left\{\begin{array}{cl}
G[k,:], k \in k_{\text {selected }} \\
0, \text { otherwise }
\end{array}\right.
$$

Where $G_{\text {matched }}$ is the CM-OFDM modulator and $k_{\text {selected }}$ is the subcarriers pattern picked out to match the channel dispersion. This selection can only be performed if CSI is available at the transmitter. So, accurate channel estimation and reliable feedback are required.

The binary $b_{s}$ bits are provided by the data source, then they are mapped on symbols to obtain $d_{s}$. After serial to parallel conversion, $d_{p}$, the $K$ non-zero complex-valued data symbols, is the input signal to the channel matched OFDM modulator to obtain $x$,

$$
x=G_{\text {matched }} d_{p}
$$

Then, parallel- serial conversion and cyclic prefix insertion are performed to obtain the transmitted signal $x_{s}$. The transmission process through the UWAC channel can be modelled by,

$$
y_{s}=x_{s} * h+n
$$

where $h$ is the channel impulse response, $n$ is additive white Gaussian noise (AWGN) with zero mean and variance $\sigma^{2}$.

\section{CM-OFDM applied in Multiuser System}

In most communication systems, multiple users must be supported. OFDM can be applied in a multiuser application, as a highly flexible and efficient communication system. The multiuser OFDM system allows the data information for several users to be transmitted on different subcarriers simultaneously. The key in the OFDM multiuser system is resource allocation to each user. The transmitters run a self-assignment algorithm to set the subcarriers, the optimal transmit power, and the 
modulation scheme for each user depending on QOS for each user and ensuring accepted system performance for all users [10].

In existing schemes, the subcarriers' assignment is uniformly or randomly distributed over the available OFDM spectrum. So, that all users experience the same channel conditions and are expected to have the same performance, assuming that they operate at the same power and rate. But in some systems, users have different priorities or some may need to transmit at higher rate or lower power so need reliable channel than others. Channel-matched subcarriers' scheduling scheme is proposed, where subcarriers characterized by high channel-transmission coefficients, low attenuation, are assigned to the users that require high QOS.

\section{Underwater Acoustic Channel Model}

Measurement analysis of acoustic propagation effects suggest that, in underwater multipath environment, the arriving signal at the receiver can be expressed as the superposition of independent copies of the transmitted signal with distinct attenuations, delays [1], [5]. Rayleigh fading channel model is suitable to model the multipath UWAC channel, where the magnitude of the amplitude of each path, $h_{l}$, has a Rayleigh distribution defined by its variance and has a probability density,

$$
p\left(h_{l}\right)=\frac{h_{l}}{\sigma_{l}^{2}} e^{\frac{-h_{l}^{2}}{2 \sigma_{l}^{2}}}
$$

Apart from multipath there are also other effects in the channel that should be modeled additionally with the chosen multipath Rayleigh model. One of those dispersive effects is Doppler Shift that is caused due to relative motion between transmitter and receiver [12]. The Doppler effect causes shift in operating frequency and is defined by,

$$
f_{l}=a_{l} f c_{l}
$$

where $f c_{l}$ is the subcarrier frequency and, $a_{l}$ is the Doppler scaling factor,

$$
a_{l}=\frac{\Delta v}{c}
$$

where $\Delta v$ is the change in relative velocity between transmitter and receiver, $c$ is the speed of sound. As the speed of sound is low compared to that of the propagated wave, this motion-induced Doppler shift can be extreme compared to the operating frequency and should be compensated at the receiver.

The time-varying frequency selective Rayleigh Fading model with Doppler effect will be considered for modelling UWAC, where the resulting linear-time-variant (LTV) channel can be described by,

$$
h(t, \tau)=\sum_{l=1}^{L} h_{l} \delta\left(\tau-\left[\tau_{l}-a_{l} t\right]\right)
$$

where $L$ is the number of channel paths, $h_{l}, \tau_{l}$, and $\mathrm{a}_{l}$ are the amplitude gain, delay, and time scale factor on the lth path, respectively [5]. 


\section{Simulation Results}

In this section, we evaluate the performance of proposed algorithm, numerical results are presented under simulated time varying frequency selective multipath channel with 20 paths. The gain of each path has Rayleigh distribution with $E\left(h_{p}{ }^{2}\right)=\frac{1}{p}, \mathrm{p}=1,2, \ldots$ L. The delays of the channel taps are uniformly distriuted with maximum delay $\mathrm{Td}=12 \mathrm{~ms}$. The channel suffers from doppler effect.We approximate the doppler shift as equal for all subcarriers and compensated by adjusting the local carrier frequency at the receiver. So, a constant doppler scaling factor, $\mathrm{a}=0.001$, is considered for all subcarriers. The OFDM system have $\mathrm{N}=128$ availale subcarriers with $f c_{\min }=15 \mathrm{kHz}$, is the minimum subcarrier frequency, $\Delta \mathrm{F}=20 \mathrm{~Hz}$, is the frequency separation between subcarriers. The duration of the OFDM symbol is denoted by $\mathrm{T}=1 / \Delta \mathrm{F}$ and the cyclic prefix has a length of $T_{c p}$, where $T_{c p}$ is always longer than the delay spread of the multipath channel, so that the OFDM symbols are ISI free [6]. Pilots are transmitted in the OFDM frame to estimate the channel at the receiver, the sucarriers used for pilots are fixed and distriuted over the whole availale bandwidth. MMSE algorithm is used for channel estimation and equalization.

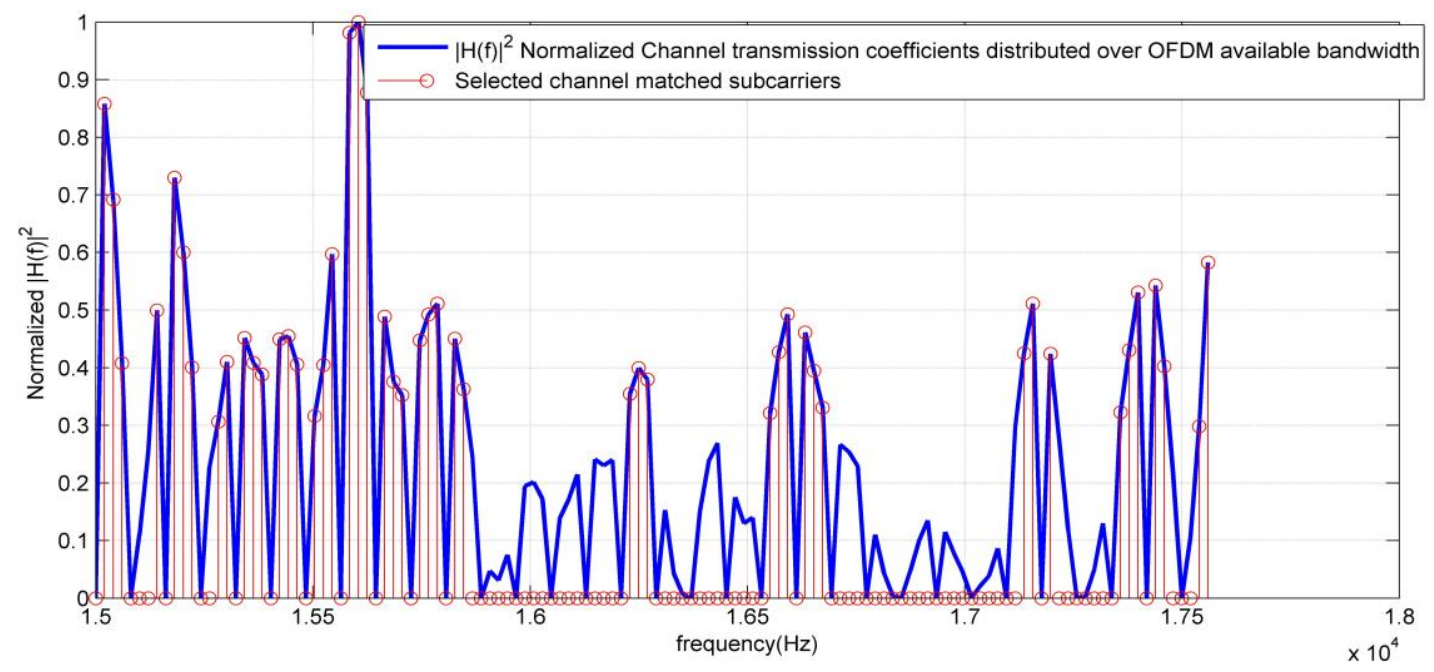

Figure 1: A representation of Selected subcarriers pattern matching the highest channel transmission coefficients in the available OFDM bandwidth.

The effect of channel-matched OFDM system is studied. We assume having 128 available frequency channels, only half data subcarriers will be used in transmission. Data is modulated using 8PSK modulator and then transmitted. The selection of subcarriers is according to the highest channel transmission coefficients, as shown in Figure 1. We compare conventional OFDM, where the subcarriers are uniformly distributed in frequency domain, and matched OFDM, where the subcarriers are selected based on channel conditions. Figure 2(a) shows that the matched algorithm has clearly improved the performance of the system in terms of probability of error. In Figure 2(b) we compare the OFDM system using all the available sub channels $(\mathrm{N}=128)$ and the adaptive OFDM using only half the sub channels. In order to have the same transmission rate, in first system data is BPSK modulated, and in the other QPSK modulation is considered. Channel-Matched OFDM still showing better 
performance than OFDM; it operates at the same rate with half spectral resources and with higher power efficiency (improved by $5 \mathrm{~dB}$ ).
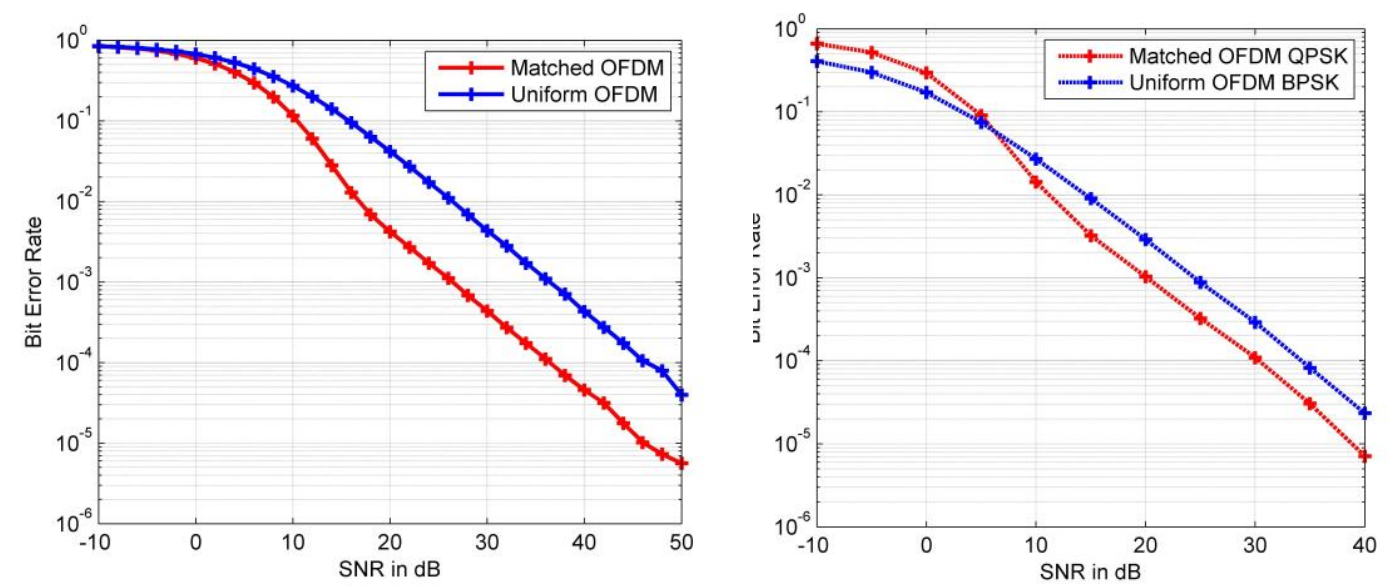

Figure 2: Comparison between OFDM and Channel-matched OFDM. (a) Both uses same number of subcarriers and same modulation, (b) OFDM with $\mathrm{N}=128$ and BPSK and CM-OFDM with N=64 and QPSK.

Then, we apply channel-matched approach to a multiuser system. For simplicity we assume only two users, each will use half of available subcarriers to transmit its data and user 1 has higher QOS. We compare different cases of resources' scheduling: random, uniform, half spectrum and the proposed scheme: in which the user with high priority will use the channel matched subcarriers and second user will assign the residual subcarriers. In Figure 3, both users transmit their data at the same rate (same modulation scheme). We compare the performance of different scheduling techniques for each user. For user1, the channel-matched subcarriers' assignment shows a significant improving in performance over the other techniques but with a slight degradation in performance of user 2 .

The power efficiency describes the ability of communication system to preserve a target bit error rate (BER). In case of user 1, power efficiency is improved by $10 \mathrm{~dB}$ while a degradation by $2.5 \mathrm{~dB}$ is noticed for user 2.

In Figure 4, user 1 works at higher rate. In normal subcarriers assignment techniques (random or uniform), user1 will suffer from lower performance than user 2, as it transmits at higher rate and at the same power. The proposed matched scheme improves the performance of user 1 with no need to increase its power to achieve the target BER. And for user 2 a slight degradation in performance is 
Channel-Matched OFDM (CM-OFDM) for Enhanced Performance of ... S. El-Khamy and D. Ibrahim

noticed, but it may be inconsiderable compared to the improving achieved for user 1 , that requires higher QOS.
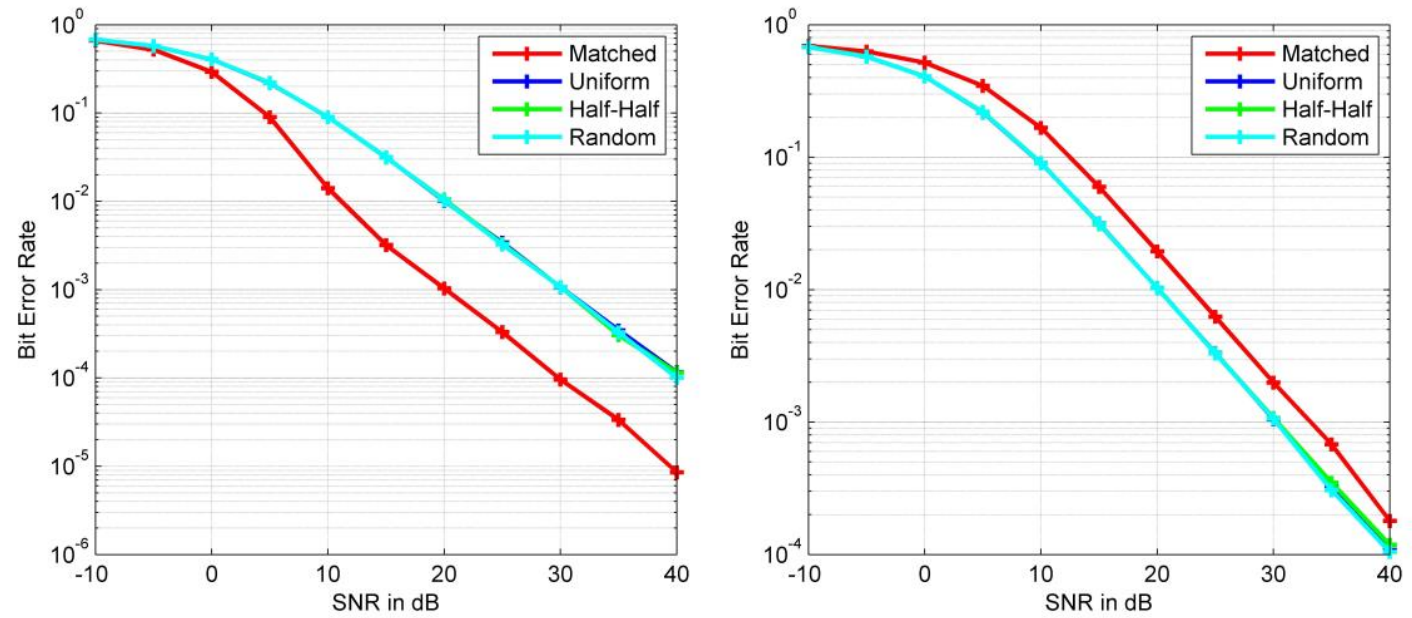

Figure 3: Comparison between different frequency assignment techniques for two-users OFDM system operating at same rate. (a)Performance of User1 has higher QOS, (b)Performance of User2.
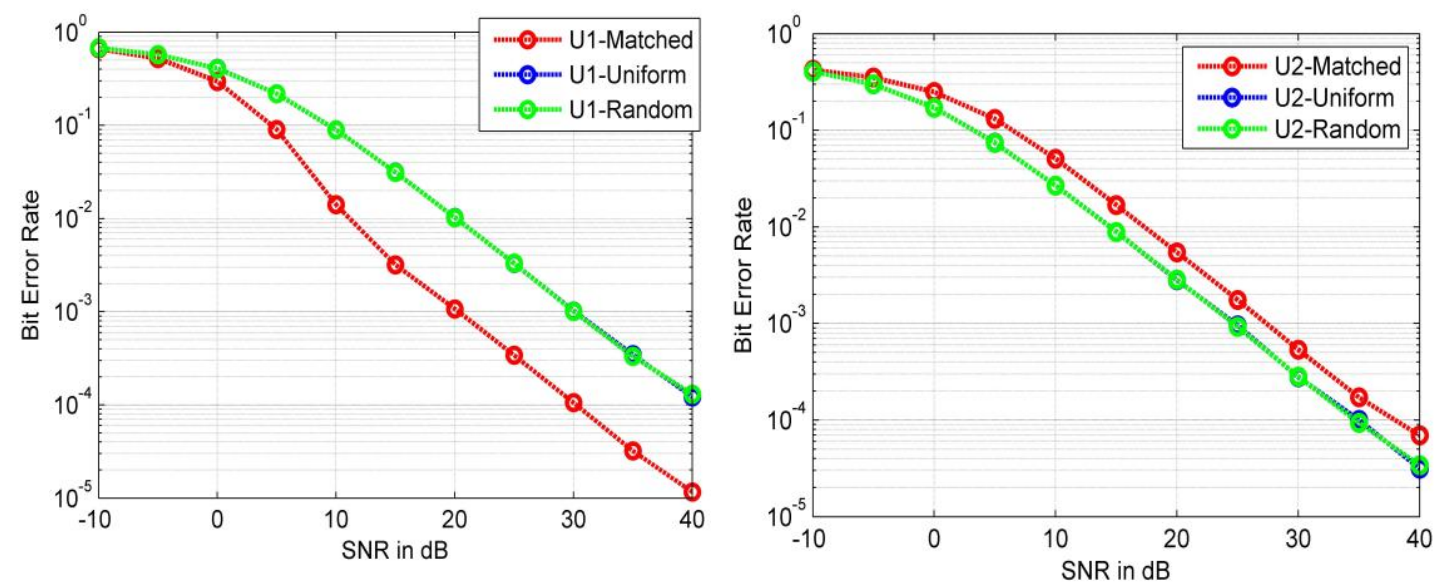

Figure 4: Comparison between different frequency assignment techniques for two-users OFDM system operating at different rate in UWAC. (a)User1 8-PSK, (b) User2 with QPSK 
We extend the work to a multiuser system with six users: user 1 requires highest QOS, then User 2, and other users have the same priorities. Figure 5 shows the performance of each user under three subcarriers' assignment techniques: random, uniform and channel-matched. The proposed scheme improves the power efficiency of users with higher QOS by $6 \mathrm{~dB}$ with unnoticeable effect on other users (degradation by $1 \mathrm{~dB}$ ).
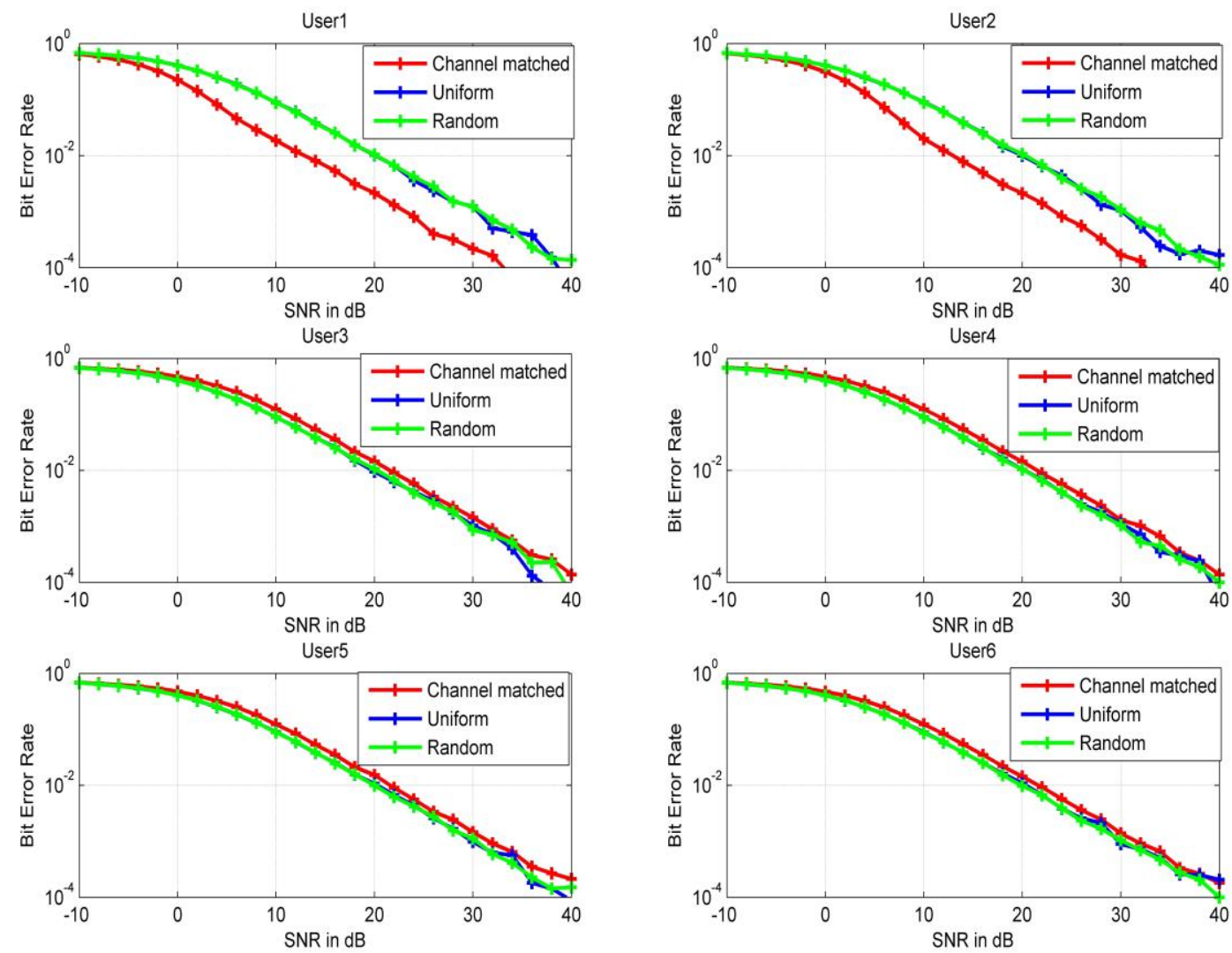

Figure 5: Performance of each user using different frequency assignment techniques for a multiuser OFDM system.

\section{Conclusion}

Channel-matched frequency hopping OFDM shows much improvement in performance compared to the conventional OFDM system in frequency selective channels. As transmission through frequency slots corresponding to deep fading, which are expected to result in high probability of error is avoided, the system throughput is improved. Taking advantage of good quality of the used channel, data can be transmitted at the same rate as OFDM, using only half spectral resources and showing better power efficiency. The unselected frequency slots can be used by another user, which may require lower QOS 
or can support difficult channel conditions. In a multiuser OFDM system, the channel-matched algorithm assign subcarriers characterized by high channel-transmission coefficients to the users that require high QOS and the remaining subcarriers are distributed over other users. Compared to other techniques, the channel-matched scheme improves significantly the performance of higher priority users. Their power efficiency is increased by $6 \mathrm{~dB}$, by adjusting the frequency pattern adaptive to channel variations and without need to increase transmitting power. And there is a little degradation of other users' performance, where power efficiency decreased by $1 \mathrm{~dB}$.

\section{References}

[1] Yun Liu, Fei Ji, Miaowen Wen, Hua Yu, Fangjiong Chen and Dehuan Wan, "Power Allocation for OFDM Over Multi-scale Multi-lag Channels", IEEE Transactions on Vehicular Technology, March 2018, Volume: 67, Issue: 3, pp. 2345 - 2358.

[2] Mohamed El-Mahallawy, Adly S. TagEldien, and Salah S. Elagooz1, "Performance Enhancement of Underwater Acoustic OFDM Communication Systems", Wireless Personal Communications, October 2019, Volume: 108, Issue: 4, pp 2047-2057.

[3] Milica Stojanovic and James Preisig, "Underwater acoustic communication channels: Propagation models and statistical characterization", IEEE Communications Magazine, January 2009, Volume: 47, Issue: 1, pp. $84-89$.

[4] Yuzhi Zhang, Yi Huang, Lei Wan, Shengli Zhou, Xiaohong Shen and Haiyan Wang, "Adaptive OFDMA with partial CSI for downlink underwater acoustic communications", Journal of Communications and Networks, June 2016, Volume: 18, Issue: 3, pp. 387 - 396.

[5] Andreja Radosevic., Rameez Ahmed, Tolga M. Duman, John G. Proakis and Milica Stojanovic, "Adaptive OFDM Modulation for Underwater Acoustic Communications: Design Considerations and Experimental Results", IEEE Journal of Oceanic Engineering, April 2014, Volume: 39, Issue: 2, pp.357-370.

[6] Y. Yao and G. B. Giannakis, "Blind carrier frequency offset estimation in SISO, MIMO, and multiuser OFDM systems," IEEE Transaction on Communications, January 2005, Volume: 53, Issue: 1, pp. 173-183.

[7] Miftahul Huda, Nisyadea Bachara Putri and Tri Budi Santoso, "OFDM System with Adaptive Modulation for Shallow Water Acoustic Channel Environment", IEEE International Conference on Communication Networks and Satellite, October 2017, pp. 55-58.

[8] Said E. El-Khamy, "Matched Frequency-Hopping (MFH) Signals for Slowly Fading Dispersive Channels", IEEE Transactions On Vehicular Technology, February 1998, Volume: 47, Issue: 1, pp. 365-369.

[9] Xiaomei Fu, Li Chen, and Jingyu Yang, "Non-orthogonal frequency division multiplexing based on sparse representation", The Institution of Engineering and Technology, October 2018, Volume: 12, Issue: 16, pp. $2005-2009$.

[10] E. Lawrey, "Multiuser OFDM", International Symposium on Signal Processing and its Applications", International Symposium on Signal Processing and its applications, August 1999, Volume: 99, pp. 761-764.

[11] Mari Carmen Domingo, "Overview of channel models for underwater wireless communication networks", Physical Communication, September 2008, Volume: 1, Issue:3, pp. 163-182.

[12] Tse, David and Pramod Viswanath, "Fundamentals of Wireless Communication", Cambridge University Press, 2005, Sec 2.4.2, pp.36. 